\title{
THE STRATEGIC AIR WAR AGAINST GERMANY AND JAPAN: A MEMOIR
}

Haywood S. Hansell, Jr.

Major General, USAF, Retired

OFFICE OF AIR FORCE HISTORY

UNITED STATES AIR FORCE

WASHINGTON, D.C., 1986 